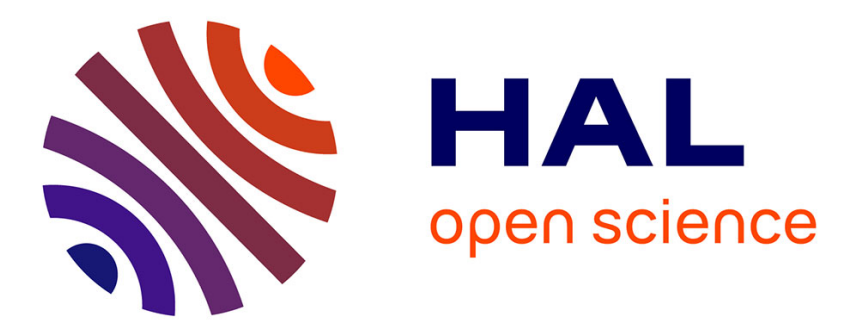

\title{
DNA double strand breaks in the acute phase after synchrotron pencilbeam irradiation
}

\author{
C. Fernandez-Palomo, E. Braeuer-Krisch, M. Trippel, C. Schroll, H. Requardt,
} S. Bartzsch, G. Nikkhah, E. Schueltke

\section{- To cite this version:}

C. Fernandez-Palomo, E. Braeuer-Krisch, M. Trippel, C. Schroll, H. Requardt, et al.. DNA double strand breaks in the acute phase after synchrotron pencilbeam irradiation. Journal of Instrumentation, 2013, 8, 9 p. 10.1088/1748-0221/8/07/C07005 . hal-01572853

\section{HAL Id: hal-01572853 \\ https://hal.science/hal-01572853}

Submitted on 8 Aug 2017

HAL is a multi-disciplinary open access archive for the deposit and dissemination of scientific research documents, whether they are published or not. The documents may come from teaching and research institutions in France or abroad, or from public or private research centers.
L'archive ouverte pluridisciplinaire HAL, est destinée au dépôt et à la diffusion de documents scientifiques de niveau recherche, publiés ou non, émanant des établissements d'enseignement et de recherche français ou étrangers, des laboratoires publics ou privés. 


\section{DNA double strand breaks in the acute phase after synchrotron pencilbeam irradiation}

\section{Fernandez-Palomo, ${ }^{a, b, 1}$ E. Bräuer-Krisch,${ }^{c}$ M. Trippel, ${ }^{a}$ C. Schroll, ${ }^{a}$ H. Requardt, ${ }^{c}$ S. Bartzsch, ${ }^{d}$ G. Nikkhah ${ }^{a}$ and E. Schültke ${ }^{a}$}

${ }^{a}$ Stereotactic Neurosurgery, Freiburg University Medical Center,

Freiburg, Germany

${ }^{b}$ Medical Physics and Applied Radiation Sciences Department, McMaster University, Hamilton, Ontario, Canada

${ }^{c}$ European Synchrotron Radiation Facility (ESRF), Grenoble, France

${ }^{d}$ Deutsches Krebsforschungszentrum (DKFZ), INF 280, Heidelberg, Germany

E-mail: fernancg@mcmaster.ca

ABSTRACT: Introduction. At the biomedical beamline of the European Synchrotron Radiation Facility (ESRF), we have established a method to study pencilbeam irradiation in-vivo in small animal models. The pencilbeam irradiation technique is based on the principle of microbeam irradiation, a concept of spatially fractionated high-dose irradiation. Using $\gamma \mathrm{H} 2 \mathrm{AX}$ as marker, we followed the development of DNA double strand breaks over $48 \mathrm{hrs}$ after whole brain irradiation with the pencilbeam technique.

Method. Almost square pencilbeams with an individual size of $51 \times 50 \mu \mathrm{m}$ were produced with an MSC collimator using a step and shoot approach, while the animals were moved vertically through the beam. The center-to-center distance (ctc) was $400 \mu \mathrm{m}$, with a peak-to-valley dose ratio (PVDR) of about 400. Five groups of healthy adult mice received peak irradiation doses of either $330 \mathrm{~Gy}$ or 2,460 Gy and valley doses of $0.82 \mathrm{~Gy}$ and $6.15 \mathrm{~Gy}$, respectively. Animals were sacrificed at 2, 12 and $48 \mathrm{hrs}$ after irradiation.

Results. DNA double strand breaks are observed in the path of the pencilbeam. The size of the damaged volume undergoes changes within the first 48 hours after irradiation.

Conclusions. The extent of DNA damage caused by pencilbeam irradiation, as assessed by H2AX antibody staining, is dose- dependent.

KEYWORDS: Radiotherapy concepts; Radiation damage evaluation methods

\footnotetext{
${ }^{1}$ Corresponding author.
} 


\section{Contents}

1 Introduction 1

2 Materials and methods $\quad 2$

2.1 Animal model and ethics statement 2

2.2 Monte Carlo calculations 2

2.3 Pencilbeam irradiation 2

2.4 Histology and immunofluorescence 3

3 Results 4

4 Discussion 5

5 Conclusion $\quad 7$

\section{Introduction}

Pencilbeam irradiation is a new concept of spatially fractionated irradiation, based on the principle of microbeam radiation therapy (MRT). Based on the dose-volume-principle, the spatial fractionation allows the application of X-ray doses in one single irradiation session that are higher by almost two orders of magnitude than X-ray doses usually applied in a single session with broad beam irradiation in the hospital environment. This should be an advantage in the therapy of highly radioresistant tumours. The technique requires a high flux of photons and therefore the availability of a synchrotrton is presently still a prerequisite.

It has been shown in studies with a classic MRT approach that the overall structure of the healthy tissue between the paths of the microbeams stays intact [1] and that the function of healthy tissue in the path of the beam is greatly preserved, possibly taking over part of the function of the tissue destroyed in the path of the microbeams [2,3]. Compared to the classic MRT approach, where the irradiated tissue is practically radiosurgically sectioned into multiple parallel slices, the three-dimensional distribution of high-dose X-ray deposits in the tissue with pencilbeam irradiation gives rise to the expectation that the tolerance of healthy brain tissue to pencilbeam irradiation might be sufficiently high to use pencilbeam irradiation for whole brain radiotherapy (WBRT).

Having previously demonstrated the feasibility of pencilbeam irradiation with the technical setup available at the biomedical beamline ID 17 of the ESRF [4], we have now designed a first experiment to study the consequences of pencilbeam irradiation on DNA integrity. 


\section{Materials and methods}

\subsection{Animal model and ethics statement}

The animals were housed and cared for in a temperature-regulated animal facility exposed to a 12-hr light/dark cycle. All experiments were performed in accordance with the guidelines of the French and German Councils on Animal Care, with permissions of the institutional committees for animal care specifically obtained at the Freiburg University Medical Center and at the ESRF.

A total of 18 healthy adult male mice (NMRI nu/nu, Charles River Germany) were used for the experiments. Of these, 15 animals were submitted to pencil beam irradiation of the entire brain, with a lateral beam propagation from right to left. Three animals served as non-irradiated controls. Of the irradiated animals, 9 were irradiated with a lower peak dose (330 Gy) and 6 animals were irradiated with a higher peak dose $(2,460 \mathrm{~Gy})$. The irradiation experiments were conducted with the animals under general anaesthesia following an intraperitoneal injection of a ketamine and xylazine cocktail (ketamine $1 \mathrm{mg} / 10 \mathrm{~g}$, xylazine $0.1 \mathrm{mg} / 10 \mathrm{~g}$ ). Animals were sacrificed at 2, 12 and $48 \mathrm{hrs}$ after irradiation ( $\mathrm{n}=3 /$ group), the brains were removed and preserved in $10 \%$ phosphatebuffered formalin.

\subsection{Monte Carlo calculations}

The general approach to Monte Carlo simulations for pencilbeam irradiation has been described previously [4]. The calculations were carried out in the Geant4 tool set (Version 4.9.3 p02) using an adjoint simulation technique. A cube of water with $16 \mathrm{~cm}$ side length was defined as target for modelling process. The reference depth was $3 \mathrm{~mm}$, corresponding to the assumed centre of the implanted tumours. Energy was scored in voxels sized $5 \times 5 \mu \mathrm{m}^{2}$ perpendicular and $4 \mathrm{~mm}$ parallel to the beam direction. At the reference depth of $3 \mathrm{~mm}$ the height was reduced to $0.5 \mathrm{~mm}$ in an additional simulation, since the dose dependency at shallow penetration depth is steep. At this depth, Monte Carlo simulations were compared to film dosimetry. With about 400 was the measured peak-to-valley dose ratio (PVDR) somewhat lower than the calculated PVDR of 530, which is most likely due to the imperfection of the model used for the measurements [4]. For safety reasons, we will continue working with the lower, measured values.

\subsection{Pencilbeam irradiation}

The irradiation experiments were conducted at ID 17, the biomedical beamline at the ESRF. The original synchrotron beam is first modified by a wiggler source, resulting in pink beam for use at the biomedical beamline. Square pencilbeams were produced using a step and shoot approach with the insertion of a multislit collimator (MSC) [5]. White synchrotron radiation X-ray beam, issued from a wiggler source, was filtered through $1 \mathrm{~mm}$ copper and $1.5 \mathrm{~mm}$ aluminum, the energy spectrum extending from about $50 \mathrm{keV}$ to well above $350 \mathrm{keV}$ with a maximum at $83 \mathrm{keV}$ [6]. The animals were placed prone on the goniometer, with the top of the skull horizontal and their teeth hooked into a small fixed holding device, and moved in vertical direction through the beam. The whole brain was irradiated in unidirectional mode, using a stop-and shoot approach during this vertical movement. Almost square pencilbeams of $50 \times 51 \mu \mathrm{m}$ were produced, the width being determined by the collimator and the hight determined by the beam slits (the horizontal beam divergence responsible for 


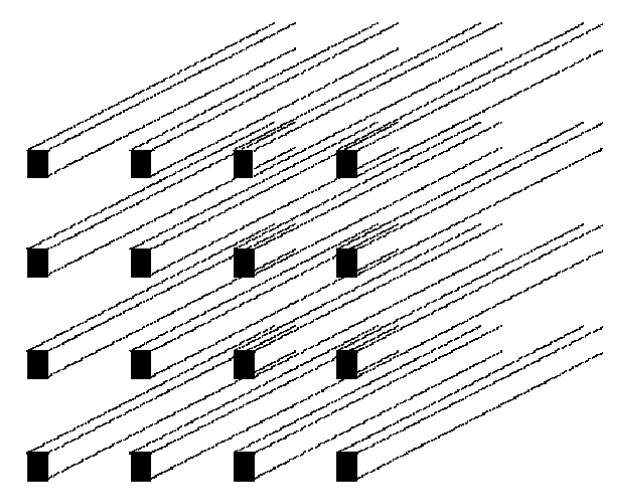

A

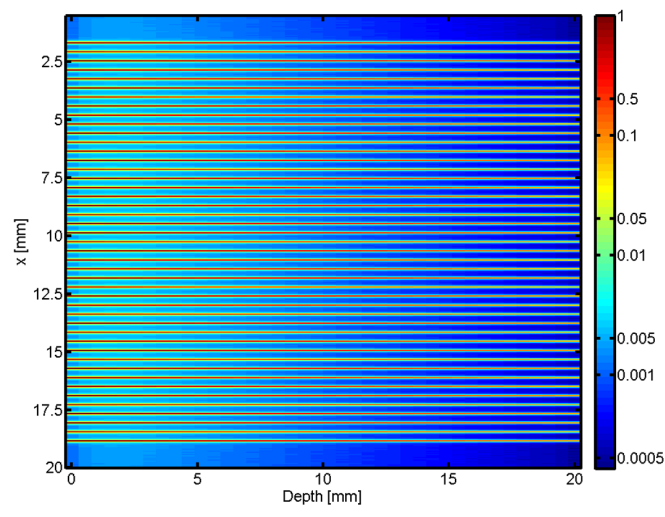

B

Figure 1. Schematic of the lateral pencilbeam profile (A) and modelling of depth dose depending on tissue depth using Monte Carlo calculations (B). Depicted is a plane in the centre of the field and parallel to the beam propagation. The valley dose is highest between 2 and $3 \mathrm{~mm}$ depth. The peak dose decreases exponentially. The beam penumbras are very narrow leaving a valley dose region of around $200 \mu \mathrm{m}$ width with constantly low dose. There are almost no differences between the beams. Valley dose decreases towards the field edges. The peak dose of the beams is almost equal at the same depth. The beam-to-beam distance is $0.4 \mathrm{~mm}$.

the 50 becoming $51 \mu \mathrm{m}$. With the right lateral side of the skull positioned orthogonally to the direction of beam propagation, the right-to-left lateral irradiation created a grid of square pencil beams (figure 1) in sagittal and an array of $50 \mu \mathrm{m}$ wide parallel stripes visible in the axial tissue sections. The animals were irradiated with a center-to-center distance (ctc) of $400 \mu \mathrm{m}$ and received peak irradiation (skin entrance) doses of either $330 \mathrm{~Gy}$ or $2460 \mathrm{~Gy}$. The irradiation pattern and the irradiation dose were recorded on Gafchromic film [7]. The irradiation times varied, depending on the ctc and total irradiation dose, between $0.035 \mathrm{sec}$ for $330 \mathrm{~Gy}$ and $0,206 \mathrm{sec}$ for 2,460 Gy. Those irradiation parameters had been chosen based on the results from our earlier feasibility study. The irradiation doses chosen for this study are slightly below the lowest and slightly above the highest doses tested in the previous experiments. Animals were sacrificed at 2, 12 and $48 \mathrm{hrs}$ after irradiation.

\subsection{Histology and immunofluorescence}

The brains were sectioned either sagittally along the midline or axially through the olfactory bulbs, placed in standard histology cassettes and stored in 10\% phosphate-buffered formalin for 7 days. They were then embedded in paraffin and $3 \mu \mathrm{m}$ thick sagittal sections were mounted on microscope slides. Histology sections were stained for Haematoxylin and Eosin (H\&E) according to a standard protocol. Immunochemistry sections were stained for fluorescence imaging with yH2AX antibody (Abcam, Cambridge, U.K.) and DAPI (Sigma). The sections were first deparaffinised and rehydrated using a series of alcohol and xylene washes. Vapour-based heat epitope retrieval was conducted by placing the slices in a pH 6 solution of citric acid at a temperature of $95^{\circ} \mathrm{C}$ for 40 minutes. Blocking of the host's proteins was performed using 5\% Goat Serum over an hour. Immediately after, the $\mathrm{yH} 2 \mathrm{AX}$ antibody was applied using a 1:100 dilution for incubation overnight at $4^{\circ} \mathrm{C}$. On the next day, the slides were rinsed 3 times with PBS and fluorochrome-conjugated secondary anti- 


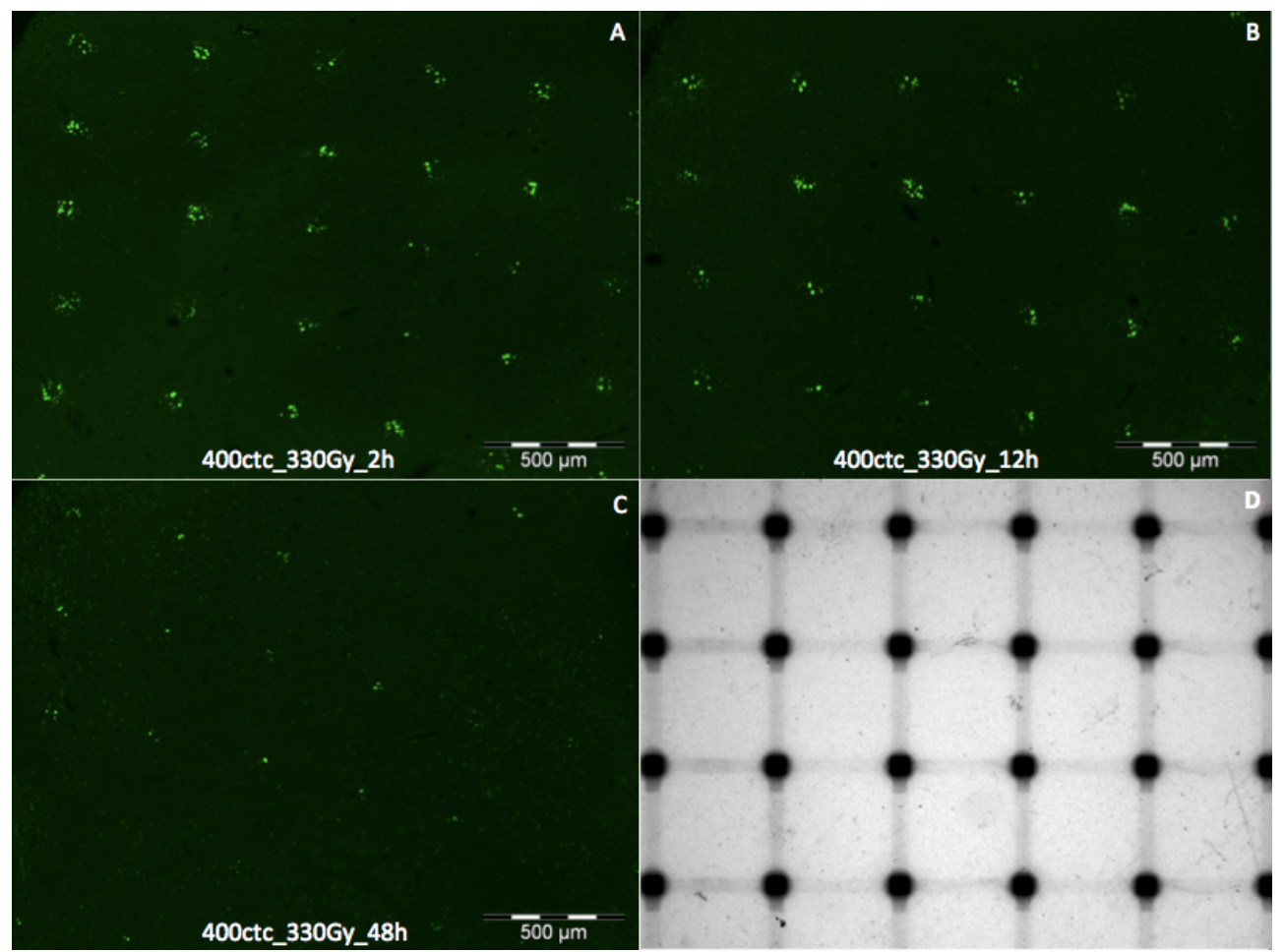

Figure 2. High magnification images, sagittal sections of mouse cortex stained with $\gamma \mathrm{H} 2 \mathrm{AX}$ antibody at 2, 12 and $48 \mathrm{hrs}$ after irradiation wit 330 Gy peak dose (A-C) and the microscopy image of a corresponding Gafchromic film (D).

body Alexa-488 (1:200 dilution) was applied over an hour at room temperature together with DAPI to counterstain the DNA. Fluorescence microscopy was conducted in the Laboratory of Molecular Neurosurgery at the Freiburg Neurocenter, at a Olympus AX 70 fluoreszence microscope.

\section{Results}

The only unexpected death occurred in the ctc $400 \mu \mathrm{m}$ high dose irradiation group. One animal irradiated with $2,460 \mathrm{~Gy}$ was found death in his cage $5 \mathrm{hrs}$ after irradiation. It was the only death occuring in this high dose irradiation group that included 14 animals, nine of them with brain tumours. However, we might have been touching the upper permissible dose limit for whole brain irradiation with a ctc of $400 \mu \mathrm{m}$.

The pattern seen in the sections stained with $\gamma \mathrm{H} 2 \mathrm{AX}$ clearly reproduced the irradiation patterns recorded on Gafchromic film (figure 2). The kinetics of the double strand breaks over time can be observed as a change of the spot diameter stained by the yH2AX stain on sagittal brain sections. The number of double strand breaks per beam spot seems to slightly increase between 2 to 12 hours and then to decrease at 48 hours after irradiation, suggesting that either DNA repair mechanisms are taking place or that the damaged cells have died and been removed by macrophages within the latter time period.

The pattern of cells with DNA double strand breaks (green) in the immunofluorescence microscopy image clearly reproduce the pattern recorded on the Gafchromic film. The dose shadow 
seen as lines that connect the pencil beam spots are originated from the horizontal slits made of polished Tungsten Carbide (50 micron) positioned just $50 \mathrm{~cm}$ prior the MSC, as well as the vertical slits used within the MSC itself. This additional dose contribution visible on the Gafchromic film is real and most likely originated from the total reflection within these slits, however the dose is orders of magnitude smaller than the peak dose within these spots from the direct beam.

The characteristic H2AX patterns were present in and around the centre of the pencilbeams at all three observation times, at two, 12 and $48 \mathrm{hrs}$ after irradiation. No double strand breaks were seen in the tissue corresponding to the centres of the dose valleys, not even after irradiation with 2,460 Gy. As expected, the area affected by DNA double strand breaks was larger after irradiation with 2,460 Gy than after irradiation with $330 \mathrm{~Gy}$. The size of the area in which DNA breaks were visible changed within the first $48 \mathrm{hrs}$ after irradiation, being largest at $12 \mathrm{hrs}$ after irradiation (figure 3). The increasing number of damaged cells over the first 12 hours is most likely due to secondary damage contributing to DNA double strand breaks. The later decrease of DNA double strand breaks can be explained by both cell repair and cell death and removal of the dead cells. While at two hours after irradiation the H2AX-positive areas appeared compact, at $12 \mathrm{hrs}$ after irradiation we noticed that the activated region became more ring-shaped.

In the sections stained with the morphological standard stain H\&E we found the characteristic dark pycnotic nuclei of cells destined for apoptosis in the area that corresponds to the centre of the beams (figure 4). This correlates with findings from classic microbeam radiation therapy, where the cells in the path of the beam become pycnotic and subsequently die.

Dark pycnotic nuclei can be seen in the centres of the radiation-induced lesions, characteristic for cells destined to undergo apoptotic cell death. The diameter of the lesion increases over time.

\section{Discussion}

Whole brain radiotherapy (WBRT) is often the last available therapeutic option for patients with multiple brain metastases. In the treatment of patients with highly malignant brain tumours, the WBRT approach has been abandoned for focal irradiation of the tumour. One of the reasons for this change of approach is the observation of changes in the white matter structure, frequently associated with cognitive and behavioural changes [8]. The therapeutic results, however, are very unsatisfactory. Patients with glioblastoma multiforme gain only a few months of survival with radiotherapy [9]. Besides radioresistance, the migration of tumour stem cells within the brain to areas outside the irradiated volume might be one of the reasons for this unsatisfactory outcome. Another reason might be that the repair capacity of tumour stem cells for damage caused by the irradiation doses applied in the hospital environment is suffciently high to allow them to survive. Spatially fractionated radiotherapy allows the application of much higher irradiation doses and therefore might be a more successful approach in the treatment of those patients.

We have shown in our experiements that the extent of DNA damage caused by pencilbeam irradiation is dose-dependent, which is in agreement with results reported by Priyadarshika and colleagues for the classsic microbeam radiation approach [10]. The development of pycnotic nuclei in the centres of the beam path corresponds to the results of our earlier experiments, where at 6 months after irradiation the centres of the beam paths were almost devoid of living cells [4]. 


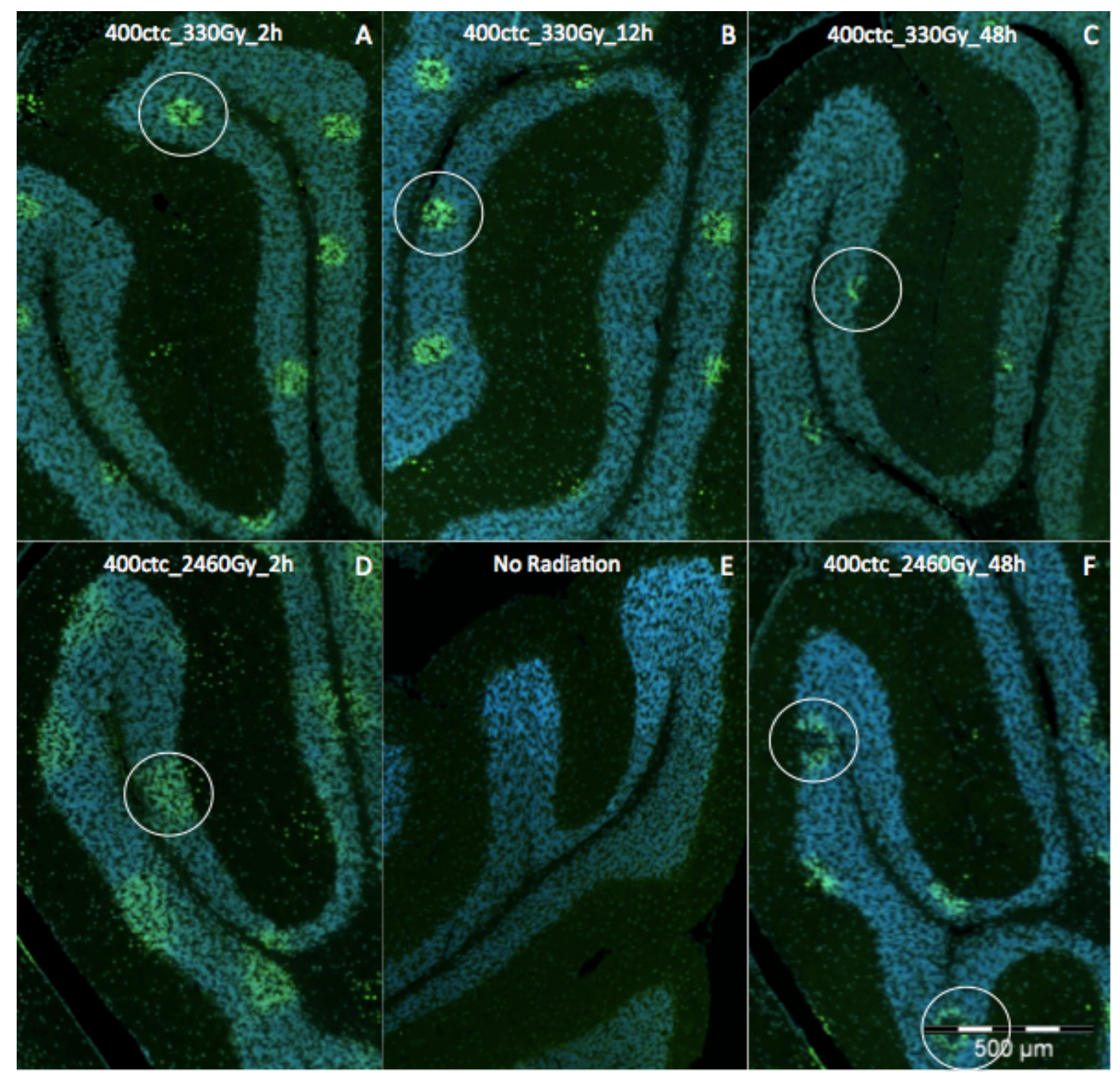

Figure 3. Low magnification images, $\gamma \mathrm{H} 2 \mathrm{AX}$ stain, sagittal sections through the cerebellum of adult mice sacrificed after irradiation with $330 \mathrm{~Gy}$ at $2 \mathrm{hrs}$ (A), $12 \mathrm{hrs}$ (B) and $48 \mathrm{hrs}$ (C), and after irradiation with 2,460 Gy at $2 \mathrm{hrs}(\mathrm{D})$, and $48 \mathrm{hrs}(\mathrm{F})$. E: section through a non-irradiated cerebellum.

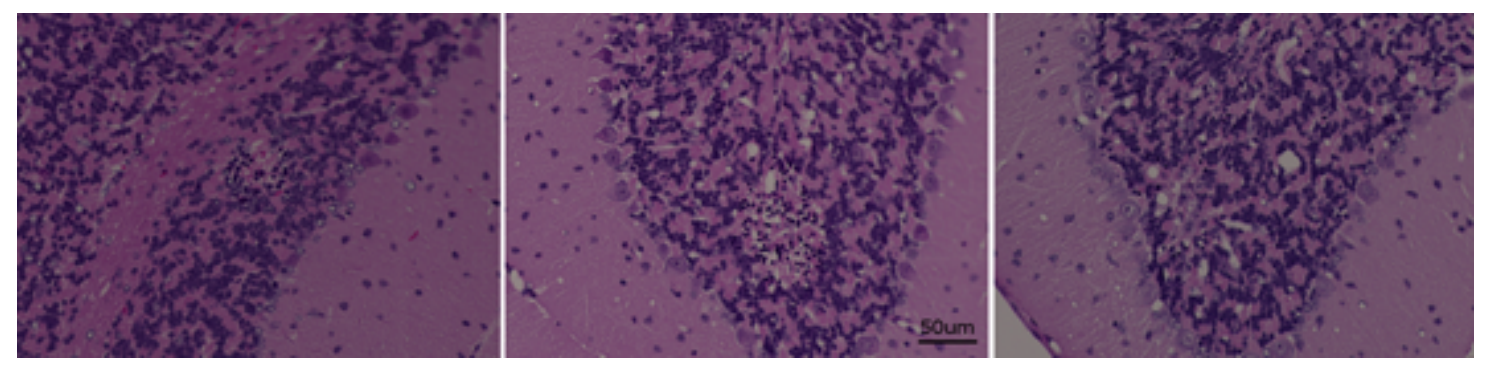

Figure 4. High magnification images, $\mathrm{H} \& \mathrm{E}$ stain of the cerebellum 2 (left), 12 (middle) and $48 \mathrm{hrs}$ (right) after whole brain irradiation with $2,460 \mathrm{~Gy}$. 
From the analysis of the H2AX study we understand that the volume in which the DNA damage occurs is not limited to the path of the beam but becomes larger than the beam in a dosedependent fashion. However, no $\gamma \mathrm{H} 2 \mathrm{AX}$-positive cells were seen in the centres of the dose valleys. In our earlier experiments we have also shown that much of the tissue between the beams remained intact, which was also reflected in memory and motor function several months after irradiation [4]. This is in agreement with results reported from work with the classic microbeam radiation approach [1-3]. From work with the classic microbeam radiation approach we also know that with some treatment protocols, a significant increase of survival times and even cures have been achieved in small animal models despite the fact that only part of the tumour volume was in the path of the microbeams [11-14]. Thus, one of the next steps will be to test in small animal models of malignant brain tumours whether pencilbeam irradiation is therapeutically effective and an increase of survival time can be achieved with the technique.

\section{Conclusion}

DNA double strand breaks as assessed by $\gamma \mathrm{H} 2 \mathrm{AX}$ antibody occur after pencilbeam irradiation in the path of the beam and in parts of the dose valleys adjacent to the path of the beam. No DNA double strand breaks occur in the centres of the dose valleys at any time between 2 and $48 \mathrm{hrs}$ after irradiation, even with doses as high as 2,460 Gy. The extent of the DNA damage is dose-dependent.

\section{Acknowledgments}

We thank Ms. Charlène Caloud from the animal facility of ID 17 (ESRF) for their dedicated animal care at the ID 17 animal facility. Dr. Geraldine Le Duc (ESRF) and Dr. Rita SanchezBrandelik (Freiburg University) and their teams are thanked for logistic support with the animal reception and transportation across international borders. We thank Dr. Alberto Bravin (ID17) for generously providing in-house research time for this project. Professor Mothersill, the mentor of Cristian Fernandez at McMaster University, is thanked for providing travel support for this student exchange. Ms. Johanna Wessolleck (Laboratory of Molecular Neurosurgery) and the technicians of the Department of Neuropathology in Freiburg are thanked for introducing Cristian Fernandez to immunofluorescence microscopy.

\section{References}

[1] J.A. Laissue et al., Neuropoathology of ablation of rat gliosarcomas and contiguous brain tissues using a microplanar beam of synchrotron-wiggler-generated x-rays, Int. J. Cancer 78 (1998) 654.

[2] E. Schültke et al., Memory and Survival after Microbeam Radiation Therapy, Eur. J. Radiol. 68 (2008) S142.

[3] J.A. Laissue et al., Weanling piglet cerebellum: a surrogate for tolerance to MRT (microbeam radiation therapy) in pediatric neuro-oncology, Proc SPIE 4508 (2001) 65.

[4] E. Schültke et al., Pencilbeam irradiation technique for whole brain radiotherapy: Technical and biological challenges in a small animal model, PLoS ONE 8 (2013) e54960. 
[5] E. Bräuer-Krisch et al., New technology enables high precision multislit collimators for microbeam radiation therapy, Rev. Sci. Instrum. 80 (2009) 074301.

[6] E. Bräuer-Krisch et al., Effects of pulsed, spatially fractionated, microscopic synchrotron X-ray beams on normal and tumoral brain tissue, Mutat. Res. 704 (2010) 160.

[7] ISP EBT Product Brief. International Speciality Products (ISP) Technologies (2007),

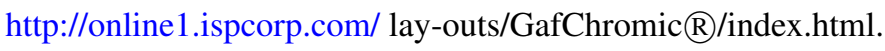

[8] J.F. Mineo et al., Prognosis factors of survival time in patients with glioblastoma multiforme: a multivariate analysis of 340 patients, Acta Neurochir. 149 (2007) 245.

[9] C.L. Armstrong, D.M. Shera, R.A. Lustig and P.C. Phillips, Phase measurement of cognitive impairment specific to radiotherapy Int. J. Oncol. Biol. Phys. 83 (2012) e319.

[10] R.C. Priyadarshika, J.C. Crosbie, B. Kumar and P.A. Rogers, Biodosimetric quantification of short-term synchrotron microbeam versus broad-beam radiation damage to mouse skin using a dermatopathological scoring system, Br. J. Radiol. 84 (2011) 833.

[11] A. Bouchet et al., Preferential effect of synchrotron microbeam radiation therapy on intracerebral $9 L$ gliosarcoma vascular networks, Int. J. Radiat. Oncol. Biol. Phys. 78 (2010) 1503.

[12] R. Serduc et al., Synchrotron microbeam radiation therapy for rat brain tumor palliation-influence of the microbeam width at constant valley dose, Phys. Med. Biol. 54 (2009) 6711.

[13] P. Regnard et al., Irradiation of intracerebral $9 L$ gliosarcoma by a single array of microplanar $x$-ray beams from a synchrotron: balance between curing and sparing, Phys. Med. Biol. 54 (2008) 861.

[14] F.A. Dilmanian et al., Response of rat intracranial $9 L$ gliosarcoma to microbeam radiation therapy, Neuro Oncol. 4 (2002) 26. 